\title{
Medicina antroposófica: um novo paradigma para as questões da medicina moderna
}

\author{
Anthroposophic medicine: a new paradigm for the questions \\ of modern medicine
}

Eliane Carapeba Rodrigues Follador

Follador ECR. Medicina antroposófica: um novo paradigma para as questões da medicina moderna / Anthroposophic medicine: a new paradigm for the questions of modern medicine. Rev Med (São Paulo). 2013 jul.-set.,92(3):166-72.

RESUMO: Nesse artigo é feita uma introdução sobre o sistema de tratamento da Medicina Antroposófica (MA), introduzidas suas bases cognitivas e científicas, os seus métodos de diagnóstico e tratamento, assim como os resultados em relação à sua situação atual, eficácia, efetividade e segurança. A MA é um sistema complementar e integrativo de tratamento que reconhece no ser humano dimensões espirituais e existenciais que interagem com os níveis somáticos e psicológicos na saúde e na doença. A MA se propõe não como uma medicina alternativa, mas como uma ampliação da prática médica pois, ao mesmo tempo que o médico antroposófico obrigatoriamente tem uma formação convencional e também lança mão dos seus recursos diagnósticos e terapêuticos, ele trabalha com os recursos trazidos por uma visão do ser humano que é provida pela Antroposofia. A MA trabalha de modo multidisciplinar, compreendendo um sistema de tratamentos que inclui medicamentos e outras modalidades de terapia. Como a MA se define como um sistema de tratamento complementar ou integrativo, são também comentados o contexto atual, as demandas e necessidades que não estão sendo adereçadas pelo modelo convencional de atendimento médico e como as práticas integrativas e complementares surgiram com uma opção para um entendimento mais amplo do processo de saúde e doença e o seu tratamento. Como um sistema de medicina integrativa, A MA se coloca como um novo paradigma para a resolução das questões que estão sendo enfrentadas atualmente, seja no âmbito existencial do indivíduo seja nas questões sociais e ecológicas da saúde. A MA tem uma proposta que pode contribuir para a ampliação do escopo de atendimento médico convencional pois tem uma base epistemológica clara, recursos próprios baseados na autonomia do paciente e mostrado bons resultados em termos de segurança, eficácia e efetividade.

DESCRITORES: Antroposofia/psicologia; Terapias complementares/psicologia; Medicina integrativa.

\begin{abstract}
This is a brief introduction to the system of Anthroposophic Medicine (AM), its cognitive and scientific foundation, its diagnostic and therapeutic approaches and information on research, safety and effectiveness. Anthroposophic Medicine is a complementary therapy system that acknowledges a spiritual and existential dimension in man, which is assumed to interact with psychological and somatic levels in health and disease. AM it is not an alternative medicine; it aims to integrate the methods, diagnosis and treatment of conventional medicine with an holistic approach to promote health and preventing and treating disease provided by Anthroposophy. AM has a multidisciplinary approach provided by physicians, and therapists and includes anthroposophic therapies (counseling, special medications, artistic and physical therapies). The treatment is individualized according to the needs of the patient. Complementary and alternative medicine has been adopted in the last decades because it appears as an answer to the needs and demands that have not been addressed by the conventional model of health care. In this scenario, AM has a new paradigm to offer in order to help address the questions faced by modern society, be it at existential level, be it the social and ecological questions. AM can contribute to the extension of conventional medical care because has an clear and well established epistemological basis that can help support the conceptual integration of complementary and conventional medicine, an approach to promote health, prevent and treat illness, and is safe, effective and can add value to the health system.
\end{abstract}

KEYWORDS: Anthroposophy/psychology; Complementary therapies/psychology; Integrative medicine.

Pesquisadora do Laboratório de Estudos em Psicologia da Arte - LAPA - Departamento de Psicologia Social e do Trabalho do Instituto de Psicologia da Universidade de São Paulo.

Endereço para correspondência: Eliane C. R. Follador. Rua Dr. Veiga Filho, 350 Cj. 808 - São Paulo, SP. CEP: 01229-000. email:efollador@gmail.com 


\section{INTRODUÇÃO}

$\mathbf{O}$ objetivo desse artigo é fazer uma introdução sobre o sistema de tratamento da Medicina Antroposófica (MA), e para isso serão discutidos brevemente suas bases cognitivas e científicas, os seus métodos de diagnóstico e tratamento, assim como os resultados em relação à sua situação atual, eficácia, efetividade e segurança.

Como a MA se define como um sistema de tratamento complementar ou integrativo, são também comentados o contexto atual, as demandas e necessidades que não estão sendo adereçadas pelo modelo de atendimento médico e como as práticas integrativas e complementares surgiram com uma opção para um entendimento mais amplo do processo de saúde e doença e o seu tratamento.

Ao discutir o paradigma no qual a medicina convencional se desenvolveu e está inserida, é colocado o conceito de pluralismo terapêutico, ou pluralismo nos cuidados da saúde, que busca o reconhecimento social, acadêmico e institucional das terapias alternativas e complementares, reforçando o consenso que a medicina convencional convive com outras formas de cuidado ${ }^{1}$.

O pluralismo médico questiona o pensamento científico dominante, mas mantém o compromisso com a ciência. Em um nível global, o pluralismo médico tem sido proposto pela Organização Mundial de Saúde (OMS), que reconhece as diversas tradições culturais de diferentes regiões do mundo ${ }^{2}$.

Finalmente, ao colocar as bases epistemológicas e filosóficas da MA, se oferece uma possibilidade de diálogo com a medicina convencional, e uma contribuição para a ampliação da visão de homem, que engloba os aspectos psicosociais e existencias biográficos, e se discute como essa abordagem pode ser uma contribuição proveitosa para o modelo atual de atendimento médico.

\section{Definição}

A MA é um sistema de tratamento que faz uso dos recursos diagnósticos e terapêuticos da medicina convencional, mas que se propõe a ampliar essa atuação a partir de uma base filosófica própria, a Antroposofia. Em consequência, provê uma visão humanista da medicina, pois trabalha a partir de uma imagem mais completa do ser humano na saúde e na doença. Esse método de diagnóstico e terapia olha para o homem como um ser que tem uma unidade de corpo, alma e espírito dentro do contexto do meio social que ele vive ${ }^{3}$.

A partir dessa fundamentação e dessa visão de mundo, a MA desenvolve seu modo de atuação e trabalha com a noção de que cada ser humano é único e deve ser tratado individualmente. $\mathrm{O}$ foco é o doente e não a doença. A vida de todo ser humano tem significado e propósito e a perda desse sentido frequentemente se reflete na deterio- ração da saúde.

Nesse contexto, considera que a doença pode prover oportunidades para mudanças positivas e para que um novo equilíbrio seja encontrado. O objetivo da Medicina Antroposófica é compreender e adereçar a multidimensionalidade do ser humano e apoiar o seu processo de desenvolvimento.

Com esse objetivo, A MA se constituiu como um sistema complementar e integrativo de tratamento que reconhece no ser humano dimensões espirituais e existenciais que interagem com os níveis somáticos e psicológicos na saúde e na doença ${ }^{4}$.

Desse modo, a MA trabalha a partir de bases teóricas próprias e de modo multidisciplinar, compreendendo um sistema de tratamentos que inclui medicamentos e outras modalidades de terapia como aconselhamento, terapias não verbais como exercícios de movimento eurítmicos, massagem rítmica, e terapias artísticas que usam pintura ou argila, música, ou exercícios da fala.

A MA se propõe não como uma medicina alternativa, mas como uma ampliação da prática médica pois, ao mesmo tempo que o médico antroposófico obrigatoriamente tem uma formação convencional e também lança mão dos seus recursos diagnósticos e terapêuticos, ele trabalha com os recurso trazidos uma visão do ser humano que é provida pela Antroposofia.

\section{Histórico}

A medicina antroposófica tem como origem o trabalho desenvolvido no começo do século XX pelo filósofo e cientista austríaco Rudolf Steiner (1861-1925).

Em 1920, a pedido de um grupo de médicos e estudantes de medicina, Rudolf Steiner desenvolveu em Dornach, na Suiça, uma série de palestras sobre doença, saúde, e a arte de curar de um ponto de vista holístico, abrangendo a existência física, emocional e espiritual pela visão da Antroposofia. A partir daí se desenvolveram as bases da MA, que resultou do empenho conjunto de um grupo de médicos liderados pela médica holandesa Ita Wegman.

A primeira clínica antroposófica foi fundada em 1921 pela Dra. Wegman, em Arlesheim, na Suíça e até hoje está em funcionamento, com uma equipe de médicos e terapeutas que trabalham com os métodos da MA para tratar principalmente doentes crônicos e pacientes com câncer. $\mathrm{Na}$ mesma época, foram criados na Suiça os laboratórios farmacêuticos Weleda e Wala.

A partir desse impulso, a MA se desenvolveu inicialmente nos países da Europa Central e se disseminou pelo mundo.

No Brasil, a MA foi introduzida há mais de 60 anos. A primeira clínica antroposófica, a Clínica Tobias, foi fundada pela médica graduada pela Faculdade de Medicina da USP, a Dra. Gudrun Burkhard, em 1969, na zona sul de São Paulo. 


\section{Bases epistemológicas}

A base teórica da MA é compatível com a abordagem hermenêutica que ajuda a compreender o ponto de vista individual dos pacientes e suas questões existenciais e espirituais ${ }^{5}$.

A MA é uma extensão da prática da medicina convencional e se baseia na visão do ser humano desenvolvida pelo filósofo austríaco Rudolf Steiner (1861-1925), fundador da Antroposofia. Uma fundamentação cognitiva e científica da Antroposofia e sua relação fundamental com as ciências naturais pode ser encontrada, entre outros, na obra literária de Rudolf Steiner ${ }^{6-8}$.

\section{Diagnóstico e abordagem terapêutica}

Na MA, o processo diagnóstico integra as habilidades técnicas e os recursos da medicina convencional a aspectos e modalidades terapêuticas desenvolvidas pela medicina antroposófica.

A MA inclui na avaliação do paciente a avaliação do equilíbrio entre o corpo e as dimensões psicológicas, mentais e espirituais. Essa abordagem busca compreender o paciente como um todo, no seu contexto emocional, mental e social e trabalhar terapeuticamente em cada um desses níveis.

Para isso, entende que a anamnese deve sempre incluir os eventos mais importantes da vida do paciente, sua biografia. A idade, a constituição, a situação biográfica e o estilo de vida do paciente são sempre levados em consideração.

\section{Trimembração e quadrimembração}

A MA amplia a visão do ser humano de um ponto de vista puramente físico para uma visão que inclui os elementos vitais, anímicos e espirituais, considerando que esses quatro elementos interagem para formar um todo, que deve ser assim considerado?.

Existe um equilíbrio delicado entre os processos que produzem consciência e que têm um efeito destrutivo, catabólico, e os processos regenerativos de construção, do elemento de vida. A partir da proposta de trimembração do homem, colocada por Rudolf Steiner em 1917, considera-se que o elemento anímico do homem se expressa pelas qualidades do pensamento, do sentimento, e da vontade, que possuem a sua base corpóreas respectivamente nos nervos, nas atividades rítmicas, e no metabolismo ${ }^{10}$.

São levados em consideração três sistemas principais, que são caracterizados pelo modo com que esses três elementos intervem no corpo físico e se baseia mais na função do que na topografia.

O sistema neurosensorial inclui todos os órgãos que surgem da camada embrionária ectodérmica: o sistema nervoso, a pele, e os órgãos dos sentidos.O sistema neu- rosensorial está principalmente localizado na cabeça, mas penetra em todo o corpo na forma dos nervos periféricos e vegetativos.

O sistema metabólicomotor inclui as funções de assimilação e nutrição, as funções do metabolismo e a atividade dos membros e dos órgãos sexuais.

O sistema rítmico compreende as funções do coração e do pulmão. Se considera que a manutenção da saúde é uma questão de manter os processos nervosos e os metabólicos, polares, em equilíbrio, e isso é feito pela intermediação do sistema rítmico.

Note-se que, no decorrer da vida, o equilíbrio muda, no início da vida ocorre domínio dos processos metabólicos e, na velhice, predominam os processos neurosensoriais.

\section{Abordagem terapêutica}

A MA atua como um sistema de tratamento que enfatiza medidas para promover a saúde e prevenir a doença. Promove programas de educação e estilo de vida e procura estimular a autonomia do paciente, trabalhando no desenvolvimento do automanejo e de coping.

$\mathrm{O}$ tratamento do paciente sempre tem um caráter direcionado, individual. A medicina ampliada pela antroposofia trata todos os aspectos do indivíduo, não apenas seus sintomas físicos, e usa a medicina ocidental convencional como sua base. O sistema de MA inclui uma abordagem ampla e multidisciplinar que permite que se empregue outras modalidades de terapia, além da medicamentosa. A partir da MA, disciplinas especializadas como aconselhamento biográfico, euritmia curativa, massagem ritmica, terapia artística e musicoterapia se desenvolveram e podem ser usadas no decorrer do tratamento.

Os remédios são derivados dos reinos animal, mineral e vegetal. Podem ser dados oralmente, por injeção ou através de aplicação externa. O tratamento visa aumentar as forças vitais do paciente, estimular os processos salutogênicos, como base para melhora da saúde e aprofundamento do auto-conhecimento ${ }^{10}$.

Faz parte do tratamento estimular o indivíduo a ser parte ativa desse processo. Como um sistema de medicina integrativa, a MA enfatiza a relação médico-paciente para apoiar os esforços do paciente para lidar com a doença, dar orientação, aumentar o otimismo, e engajar o paciente no seu processo de cura, no sentido de uma medicina salutogênica ${ }^{11}$.

A abordagem do tratamento é feita de acordo com as necessidades do paciente e lança mão de recursos de tratamento convencionais e antroposóficos. Assim, esquemas terapêuticos são raramente utilizados e o tratamento sempre é individualizado.

Por causa dessas características, na consulta médica antroposófica é feita uma história extensa, e são abordados aspectos constitucionais, psicosociais e biográficos da vida e da doença do paciente. Isso vem de encontro à valorização 
dos aspectos psicosociais do cuidado, das relações interpessoais e bem estar emocional, que impactam na qualidade de vida relacionada à saúde ${ }^{12}$.

A MA tem como objetivo tratar a doença de um modo que apóie o desenvolvimento pessoal. A terapia não almeja apenas a restauração de uma condição prévia de saúde, mas procura estimular o fortalecimento do organismo e da individualidade. Também, através do aconselhamento, os pacientes podem ter novos insights sobre suas vidas, que os ajude a fazer mudanças benéficas no seu estilo de vida. Nesse sentido, o médico trabalha ativamente com o paciente?

A importância de se adereçar o estilo de vida nas consultas tem sido cada vez mais reconhecida e considerada como um fator importante no controle de grande parte das doenças atuais. Estima-se que a maioria das doenças crônicas, que afetam 160 milhões de americanos e são responsáveis por $78 \%$ dos custos do sistema de saúde dos Estados Unidos, são causadas pelo estilo de vida e fatores do ambiente, principalmente a dieta, o sedentarismo, o tabagismo, o estresse crônico e as toxinas do ambiente ${ }^{13}$.

\section{Medicamentos}

Os medicamentos antroposóficos são produzidos de acordo com os princípios e processos farmacêuticos antroposóficos, alguns dos quais são compartilhados com a homeopatia e outros são processos não homeopáticos que refletem as interrelações entre os seres humanos e a natureza ${ }^{10}$.

Esses remédios são manufaturados de acordo com os princípios da GMP (Good Manufactoring Practice) e a sua qualidade é controlada pelos critérios e parâmetros da farmacopéia oficial.

Os medicamentos antroposóficos são prescritos em mais do que cinquenta países ao redor do mundo e se mostram seguros e custo efetivos ${ }^{15}$.

\section{A prática da MA}

A prática da MA se estabeleceu há menos de cem anos, mas tem crescido de modo consistente, não apenas na Europa central, aonde se originou, mas em várias partes do mundo.

Atualmente, encontra-se a prática da MA em mais do que 50 países ao redor do mundo, mas principalmente na Alemanha, Suiça e Holanda, aonde alcança um nível muito grande de aceitação. Na Europa, conta-se mais de 2700 médicos clínicos gerais e especialistas treinados e licenciados em MA e que praticam nos hospitais, consultórios e clínicas, e em muitos países, como na Suiça e, estão integrados no sistema de saúde.

Quano se considera a União Européia e a Suiça, são 24 hospitais (incluindo 14 com serviço de pronto socorro e dois em centros universitáros) que oferecem terapias da
MA e estima-se que 17000 médicos em prática ambulatorial prescrevam medicações da $\mathrm{MA}^{16}$.

A MA é empregada no atendimento primário e secundário em mais do que 20 países da União Européia e alcança um alto nível de satisfação entre os pacientes ${ }^{12}$.

Pesquisas têm demonstrado que a abordagem médica antroposófica é segura e efetiva e leva a uma diminuição dos gastos de seguro saúde ${ }^{4,17}$.

\section{A formação médica}

O treinamento em MA é realizado apenas para médicos formados, tem a duração de três anos, e ocorre atualmente em sete países da Europa. Fora da Europa há programas de treinamento na Argentina, Brasil, Chile, Israel, Peru, Filipinas e Estados Unidos.

Em algumas escolas de medicina, o ensino de MA faz parte do treinamento clínico em medicina integrativa. Entre 1995 e 1997 o número de escolas médicas que oferecem cursos em MA aumentou de 46 para 75 em 117 escolas médicas pesquisadas na Europa ${ }^{16}$.

\section{RESULTADOS}

Desde o seu início, a MA se desenvolveu como um sistema de terapia baseado em pesquisa. Nesse período, estudos clínicos e laboratoriais foram conduzidos de acordo com os padrões vigentes na época. Após a segunda guerra mundial, e nos anos seguintes, quando começa a se estabelecer a prática da MA na Europa central, o foco se volta para o estabelecimento da práticas, clínicas e hospitais ao invés da pesquisa ${ }^{18}$.

Nas décadas seguintes, as características dessa prática médica, centrada na relação médico-paciente, do perfil dos seus médicos e pacientes, e do uso de inúmeras possibilidades terapêuticas (medicamentosas ou não), dificultou a implementação do modelo dominante de estudos cegos randomizados. Para superar essas dificuldades, foi feito um grande esforço para desenvolver estudos em metodologia e para a atualização dos pesquisadores sobre os padrões técnicos atuais, levando em conta os mesmos critérios de qualidade e seriedade da pesquisa convencional. Isso permitiu o desenvolvimento de métodos para a realização de estudos experimentais e observacionais, o estímulo para o relato de estudos de caso, e a proposta a avaliação da MA como um sistema ${ }^{18}$.

Em 2006 foi publicada a primeira revisão sistemática sobre os a situação atual, eficácia, efetividade, segurança, utilização e custos da MA (Health Technology Assessment Report), foram pesquisadas 20 plataformas de bases de dados, e encontrados 178 pesquisas clínicas. Apesar da diferença de qualidade metodológica dos estudos, a maioria dos trabalhos mostrou bons resultados com o uso da MA, poucos efeitos colaterais, alta satisfação dos pacientes e boa relação custo/efetividade (presumivelmente pela 
diminuição de hospitalização e de uso de medicamentos) especialmente em doentes crônicos ${ }^{19}$.

Até 2009 haviam sido publicados mais do que 200 estudos clínicos sobre MA. Desses, mais da metade são sobre o uso do extrato de Viscum album no tratamento de câncer. Os efeitos clínicos dos produtos do Viscum album incluem melhora da qualidade de vida, redução dos efeitos colaterais da quimio e da radioterapia e possível aumento de sobrevida ${ }^{20,21}$.

Os resultados da aplicação da MA no tratamento de crianças também são bastante promissores. Um estudo que envolveu quatro países europeus e os Estados Unidos, e comparou o tratamento antroposófico com o tratamento convencional para infecções respiratórias agudas e otites, mostrou que pacientes tratados com o tratamento da MA apresentavam uso reduzido de antibióticos e antipiréticos, recuperação mais rápida e menos efeitos adversos ${ }^{22}$.

Outro estudo importante realizado na Suécia adereçou o estilo de vida, comparando estilo de vida antroposófico (estudantes de Escola Waldorf) com estudantes de escolas convencionais. Os autores compararam alimentação, uso de antibióticos, vacinação e o desenvolvimento de atopias. A conclusão do trabalho é que crianças com estilo de vida antroposófico apresentavam menos atopia ${ }^{23,24}$.

Quando a qualidade de vida relacionada à saúde (QVRS), que tem se tornado um parâmetro final importante, é avaliada como resultado final do estudo sobre a MA como sistema de tratamento, os resultados também são promissores, mostrando melhora clinica e estatisticamente significante e de longa duração ${ }^{17,25,26}$.

Um dos estudos mais importantes para avaliar o impacto da MA no tratamento das doenças crônicas, foi o Anthroposophic Medicine Outcomes Study (AMOS), estudo de coorte prospectivo multicêntrico conduzido entre 1998 e 2005 na Alemanha. Inicialmente, foram avaliados 392 pacientes atendidos em 90 práticas médicas (ambulatórios e consultórios).

Os primeiros resultados, após 24 meses de seguimento, mostraram melhora dos sintomas e da qualidade de vida ${ }^{17}$.

Em 2013, a publicação dos resultados com 1510 pacientes e da avaliação final de um período de seguimento de 48 meses confirmou a análise prévia, mostrando que os pacientes recebendo tratamento antroposófico para doenças crônicas mostraram melhora sustentada e clinicamente relevante dos sintomas e da qualidade de vida ${ }^{4}$.

Assim, apesar das dificuldades em se desenvolver pesquisa clínica, os esforços para o seu desenvolvimento tem conseguido mostrar de modo consistente o impacto positivo do tratamento com MA. Os principais resultados da aplicação desse sistema de tratamento se referem ao tratamento de crianças e nas doenças crônicas, onde persistentemente melhora os sintomas das doenças e a qualidade desses pacientes crônicos. A MA também mostra bons resultados para os pacientes com outras doenças, como câncer.

\section{PERSPECTIVAS FUTURAS}

Nas últimos anos aumentou o interesse pelo uso das práticas alternativas e complementares no mundo ocidental pois o reconhecimento dos enormes progressos da medicina convencional, tem sido acompanhado por uma conscientização crescente das suas limitações e dos efeitos colaterais indesejados de muitos dos seus métodos de tratamento. Há uma mudança de atitude também dos pacientes, eles já não estão dispostos a simplesmente aceitar a prescrição do médico sem entender a explicação e eles querem ter a oportunidade de discutir as opções disponíveis ${ }^{11}$.

Essa postura mais crítica em relação à medicina convencional é refletida pelo rápido aumento em popularidade das abordagens alternativas e complementares ${ }^{27}$.

Nesse panorama, se observa que o interesse e o uso das chamadas medicinas alternativas e complementares tem crescido, e muitas das universidades tem serviços de medicina integrativa e oferece cursos sobre o tema na forma de seminários e palestras.Isso é importante tanto para se certificar da qualidade dessas propostas, quanto para aumentar o conhecimento dos médicos sobre sua atuação e segurança ${ }^{11,28}$.

A Medicina Integrativa é a prática médica que reafirma a importância do relacionamento médico-paciente, foca na pessoa como um todo, é informada por evidência e faz uso de abordagens terapêuticas apropriadas, profissionais da área de saúde e disciplinas para conseguir a excelência da saúde e da cura ${ }^{29}$.

As qualidades atribuídas às medicinas integrativas são: integralidade da atenção, humanismo na relação médico-paciente, estímulo às forças curativas do organismo, menor potencial de dano e uma abordagem holística do processo saúde/doença ${ }^{1}$.

Os resultados de estudos para avaliar os resultados da MI mostram que os pacientes tratados em centros de medicina integrativa mostram melhora na qualidade de $\operatorname{vida}^{30,31}$.

No Brasil, esse interesse também ocorre, principalmente a partir dos anos 1980, quando a noção de pluralismo, que nasceu no campo das ciências políticas, começou a permear as políticas de saúde, trazendo o princípio que cidadãos socialmente iguais em direitos e deveres, podem ser diferentes em suas percepções e necessidades. Nesse período, começou a oferta de práticas complementares no SUS (homeopatia, medicina chinesa, plantas medicinais e fitoterapia e medicina antroposófica).

No Brasil, um estudo realizado em 2008 mostrou que está aumentando a aceitação das práticas integrativas e complementares pelos profissionais de saúde, sendo que, entre os profissionais pesquisados, $81,4 \%$ concordavam com a inclusão dessas práticas no SUS (Sistema Único de Saúde). Apesar desse interesse, há ainda, entre esses 
Follador ECR. Medicina antroposófica: um novo paradigma para as questões da medicina moderna.

profissionais, muito desconhecimento sobre as terapias complementares ${ }^{1}$.

O exercício da medicina antroposófica em nosso país se faz principalmente nos consultórios e clínicas, estando distante, com poucas exceções, dos centros universitários e do sistema público de saúde. Um questionário de 2004 do MS mostra que 232 municípios (11 capitais, 26 estados) ofereciam algum tipo de prática complementar em seus serviços públicos de saúde ${ }^{32}$.

A MA tem participado da política nacional de medicina natural e práticas complementares (PNMN PC) no SUS com quatro experiências de funcionamento no nível municipal do SUS: duas em Minas Gerais (São João del Rey e Belo Horizonte), Rio de Janeiro e em São Paulo ${ }^{33}$.

\section{CONCLUSÃO}

O ensino médico atual é pensado a partir de um ponto de vista muito específico, que olha para as coisas de um ponto de vista biotecnológico. Para compreender o momento atual e as demandas tanto do ensino médico quanto da formulação das políticas públicas de saúde, é de fundamental importância tomar consciência sobre as bases epistemológicas que norteiam as nossas decisões, pois isso nos ajuda a perceber a sua relatividade.

Nesse sentido, o conhecimento da história da medicina nos permite conhecer outros pontos de vista sobre a saúde, a doença e a cura e ver como isso se relaciona conosco. Isso nos ajuda a perceber como evoluiu o pensamento e a consciência humana no decorrer do tempo e que

\section{REFERÊNCIAS}

1. Thiago SCS, Tesser CD. Percepção de médicos e enfermeiros da estratégia de saúde da família sobre terapias complementares. Rev Saúde Pública. 2011;45(2):249-57. http://dx.doi. org/10.1590/S0034-89102011005000002.

2. Kiene H, Brinkhaus B, Fischer G, Hahn EG, Hoppe HD, Jütte R, Kraft K, Klitzch W, Mathiessen PF, Meister P, Michalsen A, Teut M, Willich SN, Heimpel H. Professional treatment in the context of medical pluralism - A German perspective. Eur J Integr Med. 2010;2:53-6. http://dx.doi.org/10.1016/j. eujim.2010.04.002

3. Bott V. An introduction to anthroposophic medicine. Extending the art of healing. 2nd ed. Forest Row: Sophia Books; 2004.

4. Hamre HJ, Kiene H, Glockman A, Ziegler R, Kienle GS. Long-term outcomes of anthroposophic treatment for chronic disease: a four-year follow-up analysis of 1510 patients from a prospective observational study in routine outpatient settings. BMC Res Notes. 2013;6:269. Available from: http://www. biomedcentral.com/1756-0500/6/269.

5. Heusser P. A basis for the understanding of anthroposophical medicine and cancer therapy. Trad. Harold Jurgens. Chestnut Ridge, NY: Mercury Press; 1991. Available from: http://www. direção queremos tomar. E é esse conhecimento que pode nos ajudar a colocar em perspectiva as nossas idéias e ver a sua relação com o outro.

É importante lembrar que uma ciência médica sem uma visão de mundo, na verdade é impossível, cada método terapêutico pressupõe conhecimento específico, uma visão própria da natureza e do homem. A questão não é discutir a visão de mundo, mas se é possível estabelecer de modo científico a sua aplicação prática ${ }^{5}$.

Além disso, o aumento de conhecimento dos médicos sobre outros sistemas alternativos e complementares assegura a segurança do uso dessas práticas complementares e a incorporação dos benefícios associados à abordagem da $\mathrm{MI}^{34}$.

O pluralismo e o diálogo são princípios chave para sociedades abertas e tolerantes. O conceito de pluralismo, surgiu na teoria da ciência no final do século XX com o reconhecimento da estrutura pluralística da ciência. De modo similar, um sistema de sistema pluralista, apoiado por um discurso crítico contínuo e pesquisa científica, é a resposta apropriada para o tratamento das múltiplas formas e complexidades das doenças.

Nesse sentido, a MA se apresenta como uma prática médica integrativa, e que pode contribuir para a ampliação do escopo de atendimento médico convencional, contribuindo para as mudanças de paradigma que atendam às demandas do cuidado em saúde e às necessidades dos pacientes, pois tem uma base epistemológica clara, recursos próprios baseados na autonomia do paciente e tem mostrado bons resultados em termos de segurança, eficácia e efetividade.

anthromed.org/Article.aspx?artpk=716

6. Steiner R. Goethean science. Chestnut Ridge, NY: Mercury Press; 1988

7. Steiner R. Filosofia da liberdade - Fundamentos para uma filosofia moderna. 4a ed. São Paulo: Editora Antroposófica; 2008 .

8. Steiner R, Wegman I. Elementos fundamentais para uma ampliação da arte de curar. 3a ed. São Paulo: Editora Antroposófica; 2007.

9. Evans M, Rodger I. Anthroposophical medicine. Healing for body, soul and spirit. London: Thorsons; 1992.

10. Vogel H-H, McKibbon M. Finding remedies. Spiritual knowledge of man and nature. Berlin: Natur Mensch Medizin Verlags; 2000.

11. Kienle GS, Albonico HU, Fischer L, Frei-Erb M, Hamre HJ, Heusser P, Mathiessen P, Renfer A, Kiene H. Complementary therapy systems and their integrative evaluation. Explore (NY). 2011;7(3):175-87. doi: 10.1016/j.explore.2011.02.001.

12. Esch BM, Busato A, Heusser P. Patient satisfaction with 
primary care: an observational study comparing anthroposophic and conventional care. Health Qual Life Outcomes. 2008;6:24. Available from: http://www.hqlo.com/content/pdf/1477-75256-24.pdf. doi:10.1186/1477-7525-6-24

13. Ryan MA, Ornish D, Roizen M. Lifestyle medicine: treating the causes of diseases. Altern Ther Health Med. 2009;15(6):12-4. Available from: http://drhyman.com/downloads/LifestyleMedicine.pdf

14. International Federation of Anthroposophic Medical Associations (IVAA). The system of anthroposophic medicine. Brussels: IVAA; 2011. Available from: http://www.ahasc.org. uk/media/15717/system_anthroposophic_medicine_print.pdf

15. Hamre HJ, Witt CM, Glockmann A, Tröger W, Willich SN, Kiene H. Use and safety of anthroposophic medications in chronic disease: a four year prospective cohort study. Drug Safety. 2006;29:12:1173-89. DOI:10.2165/00002018200629120-00008

16. Hamre Zimmerman P, Winkler M, Arendt A, Bender A, Breitkreutz T, Glöekler M, et al. Facts and figures on anthroposophic medicine worldwide. Brussels: International Federation of Anthroposophic Medical Associations; 2012. Available from: http:/www.ivaa.info/fileadmin/editor/file/ Facts_and_Figures_AM_WorldwideJuly2012_Final_Public_ Light.pdf

17. Hamre HJ, Becker-Witt C, Glockman A, Ziegler R, Willich $\mathrm{SN}$, Kiene H. Anthroposophic therapies in chronic disease: the Anthroposophic medicine outcomes study (AMOS). Eur J Med Res. 2004;9:351-60. Available from: http://www.ifaemm. de/Abstract/PDFs/HH04_2.pdf

18. Hamre HJ, Kiene H, Kienle GS. Clinical research in anthroposophic medicine. Altern Ther Health Med. 2009;15(6):52-5. Available from: http://web.a.ebscohost.com/ ehost/pdfviewer/pdfviewer?sid=2bdd583a-c423-4cb4-a157-2 5a62f1 f0408\%40sessionmgr4001\&vid=2\&hid=4209

19. Kienle GS, Kiene H, Albonico H: Anthroposophische Medizin in der klinischen Forschung. Wirksamkeit, Nutzen, Wirtschaftlichkeit, Sicherheit. Stuttgart: Schattauer; 2006.

20. Kienle GS, Kiene H. Complementary cancer therapy: a systematic review of prospective clinical trials on Anthroposophic mistletoe extracts. Eur J Med Res. 2007;12 (3):103-19. Available from: http://www.ifaemm.de/Abstract/ PDFs/GK07_1.pdf

21. Kienle GS, Kiene H. Influence of viscum album 1 (European mistletoe) extracts on Quality of life in cancer patients: A systematic review of controlled clinical studies. Integr Cancer Ther. 2010;9(2):142-57. Available from: http://ict.sagepub.com/ content/9/2/142.full.pdf. DOI: 10.1177/1534735410369673

22. Hamre HJ, Fischer M, Heger M, Riley D, Haidvogl M, Baars E, Bristol E, Evans M, Schwarz R, Kiene H. Anthroposophic vs. conventional therapy of acute respiratory and ear infections: a prospective outcomes study. Wiener Klinische Wochenschrift. 2005;117(7/8):256-68. http://dx.doi.org/10.1007/s00508-0050344-9

23. Alm JS, Swartz J, Lilja G, Scheynius A, Pershagen GJ. Atopy in children of families with an anthroposophical lifestyle. Lancet. 1999;353:1485-8. Available from: http://www.whale. to/vaccine/rb5103.pdf

24. Flöistrup H, Swartz J, Bergström A, Alm JS, Scheynius A, van Hage M, Waser M, Braun-Fahrländer C, Schram-Bijkerk D, Huber M, Zutavern A, von Mutius E, Üblagger E, Riedler J, Michaels KB, Pershagen G. PARSIFAL Study Group. Allergic disease and sensitization in Steiner school children. J Allergy Clin Immunol. 2006;117(1):59-66. http://dx.doi.org/10.1016/j. jaci.2005.09.039

25. Carlsson M, Arman M, Backman M, Hamrin E. Perceived quality of life and coping for Swedish women with breast cancer who choose complementary medicine. Cancer Nurs. 2001;24(5):395-401. doi:10.1097/00002820-20011000000012

26. Heusser P, Berger Braun S, Bertschy M, Burkhard R, Ziegler R, Helwig S, van Wegberg B, Cerny T. Palliative in-patient cancer treatment in an anthroposophic hospital: II. quality of life during and after stationary treatment, and subjective treatment benefits. Forsch Komplement Med. 2006;13(3):15666. doi:10.1159/000092448

27. Quandt SA, Verhoef MJ, Arcury TA, Lewith GT, Steinsbeck A, Kristoffersen AE, Wahner-Roedler DL, Fonnebo V. Development of an International Questionnaire to measure use of complementary and alternative medicine (I-CAM-Q). J Altern Complement Med. 2009;15(4):331-9. doi: 10.1089/ acm.2008.0521.

28. Nicolao M, Tauber MG, Heusser P. How should complementary and alternative medicine be taught to medical students in Switzerland? A survey of medical experts and students. Med Teacher. 2010;32(1):50-5. doi: $10.3109 / 01421590902825123$

29. Heusser P. Integrative medicine and the quest for humanism in medicine [editorial]. Forsch Komplemented. 2011;18:172-3. doi: $10.1159 / 000330650$

30. Greeson JM, Rosenzweig S, Halbert, SC, Cantor IS, Keener MT, Brainard GC. Integrative medicine research at an academic medical center: patients characteristics and health related quality of life outcomes. J Altern Complement Med. 2008;14(6):763-7. doi: 10.1089/acm.2008.0114

31. Ostermann T, Langhorst J, Beer, A-M. The effects of integrative in-patients treatment on patients' quality of life: a meta-analysis. Evidence Based Complement Altern Med. 2013;2013:416510. doi: 10.1155/2013/416510.

32. Barros NF, Siegel P, De Simoni C. Política nacional de práticas alternativas e complementares no SUS-PNPIC-SUS. Cad Saúde Pública, Rio de Janeiro. 2007;23(12):3066-9. Disponível em: http://bvsms.saude.gov.br/bvs/publicacoes/pnpic.pdf

33. Benevides IA. Relato de desenvolvimento de política de medicina natural e práticas complementares. Art Med Ampl. 2004;24(3/4):38-40

34. Ben-Arye E, Schiff E, Zollman C, Heusser P, Mountford P, Frenkel M, Bar-Sela G, Lavie O. Integrating complementary medicine in supportive cancer care models across four continents. Med Oncol. 2013, 30:511. doi: 10.1007/s12032013-0511-1. 